Andrew M. Bouwma · Kenneth J. Howard •

Robert L. Jeanne

\title{
Parasitism in a social wasp: effect of gregarines on foraging behavior, colony productivity, and adult mortality
}

While the HTML version of this article is correct, the PDF and the print versions show errors, for which we sincerely apologize.

The reference "Schmid Hempel" should always read "Schmid-Hempel."

On page 224 in the section, "Foraging-rate study," fourth paragraph, the fourth sentence must read:

"P. occidentalis forages for nest materials (pulp or water) and food (nectar or prey) (O'Donnell and Jeanne 1990)."

On pages 225-226 in the section, "Colony productivity," the second and third paragraphs must read:

"In a multiple weighted regression (weighted by $A$ squared), using a backwards elimination procedure, we fitted cells per capita $\left(C_{\mathrm{pc}}\right)$ on infection prevalence $\left(G_{\mathrm{prev}}\right)$, total adults in the colony on day $25(A)$, the proportion of queens in the colony $(Q)$, infection prevalence in queens $\left(Q_{\text {prev }}\right)$, collection date $(D)$, a class variable for year $(Y)$, and interaction terms $\left(G_{\text {prev }} \times Y, A \times Y, Q \times Y, Q_{\text {prev }} \times Y\right.$, and $D \times Y)(N=13$ for 1998,41 for 1999 , and 12 for 2000). We weighted by $A$-squared because inspection of residual plots indicated greater variance in $C_{\mathrm{pc}}$ for small values of $A$ (Chatterjee et al. 2000).

In a second multiple regression, we fitted brood weight $(\mathrm{mg})$ per capita $\left(B_{\mathrm{pc}}\right)$ on the aforementioned independent variables $(N=13$ for 1998, 40 for 1999, and 0 for 2000). We did not weight by $A$-squared for this model because variance was constant. We used PROC GLM in SAS version 8.2 for all multiple regression analyses."

The online version of the original article can be found at http://dx. doi.org/10.1007/s00265-005-0028-5.

A. M. Bouwma $(\bowtie) \cdot$ R. L. Jeanne

Department of Entomology, University of Wisconsin, Madison, WI 53706, USA

e-mail: bouwma@entomology.wisc.edu

Tel.: +1-608-265-6128

Fax: +1-608-262-3322

K. J. Howard

Department of Zoology, University of Wisconsin,

Madison, WI 53706, USA 